\title{
CORROSÃO POR PITE DO AÇO INOXIDÁVEL DUPLEX UNS S32205 LAMINADO A MORNO*
}

\section{Resumo}

\author{
Raphael França Assumpção ${ }^{1}$ \\ Daniela Barçante Perasoli \\ Davi Silva Alves ${ }^{2}$ \\ Aline Oliveira Vasconcelos Ferreira ${ }^{2}$ \\ lane Dutra Moutinho ${ }^{2}$ \\ Indiana Rosa Oliveira ${ }^{2}$ \\ Dagoberto Brandão Santos ${ }^{3}$ \\ Dalila Chaves Sicupira ${ }^{4}$
}

O aço inoxidável duplex (AID) vem sendo considerado uma excelente alternativa para aplicações que requerem alta resistência à corrosão e alta resistência mecânica. Portanto, a microestrutura e as propriedades dos aços dúplex permanecem como temas de grande interesse. Para este proposito, estudou-se o efeito da laminação a morno em um aço inoxidável duplex. O foco deste trabalho foi o AID 2205 que é um dos tipos mais comuns de AID. O objetivo deste estudo é avaliar o comportamento eletroquímico do AID 2205 laminado a morno no ambiente de íons de cloretos utilizando medidas de polarização potenciodinâmica. O aço inoxidável dúplex foi laminado a morno à $600^{\circ} \mathrm{C}$ para 60 e $80 \%$ de redução na espessura e submetido a testes eletroquímicos. As amostras apresentaram uma excelente resistência à corrosão em solução de $\mathrm{NaCl}$ $3,5 \%$. Apesar da existência de certa similaridade entre os valores de potencial de circuito aberto e potenciais de pites obtidos para todas as amostras, o número de pites observado foi maior na amostra CF, seguida pelas amostras com 60 e $80 \%$ de redução.

Palavras-chave: Corrosão por pites; Aço inoxidável Duplex; Tratamento termomecânico; Polarização cíclica.

\section{PITTING CORROSION OF WARM ROLLED UNS S2205 DUPLEX STAINLESS STEEL Abstract}

Duplex stainless steel (DSS) has been considered as an excellent alternative for applications where high corrosion resistance and high mechanical strength are required. Therefore, microstructure and properties of duplex steels remain topics of much interest. For this purpose, the effect of warm-rolling was studied in a duplex stainless steel. The focus of this work was 2205 DSS which is one of the most common types of DSS. The objective of this study is to evaluate the electrochemical behavior of warm-rolled 2205 DSS in the chlorine ion environment using potentiodynamic polarization measurements. The duplex stainless steel was warm-rolled at $600^{\circ} \mathrm{C}$ to 60 and $80 \%$ reduction in thickness and submitted to electrochemical tests. The samples exhibited an excellent corrosion resistance in $3.5 \% \mathrm{NaCl}$ solution. Despite the existence of a certain similarity among the values of open circuit potential and pitting potentials obtained for all samples, the number of pits observed was higher in the CF sample, followed by the samples with 60 and $80 \%$ reduction.

Keywords: Pitting Corrosion; Duplex Stainless Steel; Thermomechanical Treatment; Cyclic polarization.

1 Engenharia Metalúrgica, Graduando, Estudante, Departamento de Engenharia Metalúrgica e de Materiais, Universidade Federal de Ouro Preto, Ouro Preto, MG, Brasil.

2 Engenharia Metalúrgica, Graduando, Estudante, Departamento de Engenharia Metalúrgica e de Materiais, Universidade Federal de Minas Gerais, Belo Horizonte, MG, Brasil.

3 Engenharia Metalúrgica, Doutor, professor, Departamento de Engenharia Metalúrgica e de Materiais, Universidade Federal de Minas Gerais, Belo Horizonte, MG, Brasil.

4 Química, Doutora, Professora, Departamento de Química, Universidade Federal de Ouro Preto, Ouro Preto, MG, Brasil. 


\section{INTRODUÇÃO}

Os aços inoxidáveis duplex (AID) combinam propriedades das fases austenita e ferrita e são caracterizados como materiais com bom desempenho sob altas cargas mecânicas e resistentes à corrosão, especialmente em condições de corrosão sob tensão em ambientes contendo cloretos [1]. São ligas bifásicas baseadas no sistema Fe-Cr-Ni-Mo nas quais as proporções dos elementos constituintes permitem a otimização do equilíbrio das frações volumétricas de austenita e ferrita [2].

O número de aplicações para o aço inoxidável duplex aumenta de forma constante e novas ligas são continuamente desenvolvidas devida a volatilidade do preço do $\mathrm{Ni}$. Nos AID o níquel contido é parcialmente substituído por manganês e nitrogênio, e, além disso, o teor de molibdênio é reduzido [3].

As propriedades desses aços lhe permitem diversas aplicações como mineração, caldeiras, papel, química, petroquímica e indústrias offshore. A concentração mais baixa de níquel tornou vantajosa sua utilização como um biomaterial no corpo humano. Os AID vem sendo usados também nas indústrias de alimentos e farmacêuticas [1,4].

A microestrutura constituída de austenita e ferrita em quantidades aproximadamente iguais e a ausência de fases secundárias prejudiciais tais como a fase $\sigma$ e nitreto de cromo, $\mathrm{Cr}_{2} \mathrm{~N}$, proporcionam melhora das propriedades mecânicas, juntamente com a resistência à corrosão em comparação com os habituais aços inoxidáveis monofásicos, ferríticos ou austeníticos $[5,6,7]$.

A elevada resistência à corrosão dos aços inoxidáveis duplex é devida a elementos de liga tais como $\mathrm{Cr}$, Mo e $\mathrm{N}$. No entanto, a resistência à corrosão desses aços inoxidáveis é altamente dependente da microestrutura, que é afetada pelo tratamento termomecânico [8,9]. Durante o processamento termomecânico, tal como a laminação a morno, esta liga é exposta a temperaturas elevadas que causam mudanças microestruturais na mesma.

Embora o processo de laminação possa ser realizado a temperaturas acima da metade do ponto de fusão do metal, denominado laminação a quente, ou abaixo dessa temperatura, caso em que se trata de laminação a frio. Há uma faixa de temperatura, começando abaixo e terminando acima da linha divisória entre laminação a quente e a frio, dentro da qual o processo é denominado laminação a morno [10]. Estes processos levam a alterações mecânicas e metalúrgicas dos atributos da peça de trabalho, que não são possíveis de se conseguir nos regimes a frio ou a quente [10].

Do ponto de vista comercial, a laminação a morno é considerado particularmente benéfica na realização de novos produtos e na redução no custo de produção de tiras laminadas. O efeito da laminação a morno em AIDs é considerado interessante, particularmente devido ao fato de que a ferrita e a austenita poderem apresentar diferentes comportamentos de amaciamento durante a deformação. Tem-se argumentado que as duas fases se deformam de forma independente, e a evolução da microestrutura e textura pode ser inferida a partir do comportamento de materiais monofásicos [5,11].

Até o momento, não há estudo sobre o efeito da laminação a morno do aço inoxidável duplex 2205 na sua resistência à corrosão. Neste contexto, o objetivo desse trabalho é avaliar a resistência à corrosão do aço inoxidável duplex UNS S32205, após a etapa de laminação a morno, em meio contendo íons cloreto.

\section{MATERIAIS E MÉTODOS}


O material em estudo foi laminado a morno a $600^{\circ} \mathrm{C}$ com reduções de 60 e $80 \%$ de espessura. O aço UNS S32205 foi fornecido pela Aperam South America na forma de chapas laminadas a quente com $5,5 \mathrm{~mm}$ de espessura, $150 \mathrm{~mm}$ de comprimento e $100 \mathrm{~mm}$ de largura. A composição química do aço usado neste trabalho é apresentada na Tabela 1.

Tabela 1. Composição química (\% em massa) do aço inoxidável UNS S32205

\begin{tabular}{lccccccccc}
\hline Elemento & $\mathbf{C}$ & $\mathbf{M n}$ & $\mathbf{S i}$ & $\mathbf{C r}$ & $\mathbf{N i}$ & $\mathbf{M o}$ & $\mathbf{N}$ & $\mathbf{P}$ & $\mathbf{S}$ \\
\hline \% em massa & 0,026 & 1,83 & 0,25 & 22,43 & 5,44 & 3,04 & 0,15 & 0,03 & 0,0002 \\
\hline
\end{tabular}

A resistência à corrosão por pite de cada condição foi avaliada utilizando a técnica de polarização potenciodinâmica cíclica em solução de $\mathrm{NaCl} 3,5 \%$ à temperatura ambiente. Os testes de polarização cíclica foram realizados em uma célula convencional de três eletrodos utilizando uma placa de platina como eletrodo auxiliar e um eletrodo $\mathrm{Ag} / \mathrm{AgCl}$ como eletrodo de referência. O equipamento utilizado foi um potenciostato da marca Autolab, modelo PGSTAT302N. Os testes foram realizados segundo a Norma ASTM G 61 [12].

O eletrodo de trabalho foi construído utilizando chapas de AID que foram cortadas com dimensões de $10 \times 10 \mathrm{~mm}$ e embutidas em resina acrílica. O contato elétrico foi estabelecido soldando-se um fio metálico na superfície da amostra. Antes de cada experimento, os eletrodos de trabalho foram lixados utilizando as lixas de 100, 240, 320, 400, 600 mesh, desengordurados com detergente, álcool e lavados em água.

A polarização foi iniciada após 60 min de estabilização do potencial de circuito aberto $(O C P)$. Aplicou-se uma taxa de varredura na direção anódica de $0,167 \mathrm{mV} / \mathrm{s}$ até se atingir uma densidade de corrente de $1 \mathrm{~mA} / \mathrm{cm}^{2}$. Neste ponto a varredura foi revertida para direção catódica até 0 valor de $O C P$ ser atingido. Os testes eletroquímicos foram realizados em triplicata. A caracterização dos pites após o teste eletroquímico foi feita através da técnica de microscopia eletrônica de varredura (MEV).

\section{RESULTADOS E DISCUSSÃO}

As Figuras 1 e 2 apresentam, respectivamente, os potenciais de circuito aberto e as curvas de polarização potenciodinâmica cíclica obtidas para amostras de aço 2205 como fornecido (CF) e após processo de laminação à morno, com $60 \%$ e $80 \%$ de redução. Os valores médios de OCP, potenciais de repassivação $\left(E_{\text {rep }}\right)$ e de pites $\left(E_{\text {pit }}\right.$ e os respectivos desvios padrão estão resumidos na Tabela 2 abaixo. 


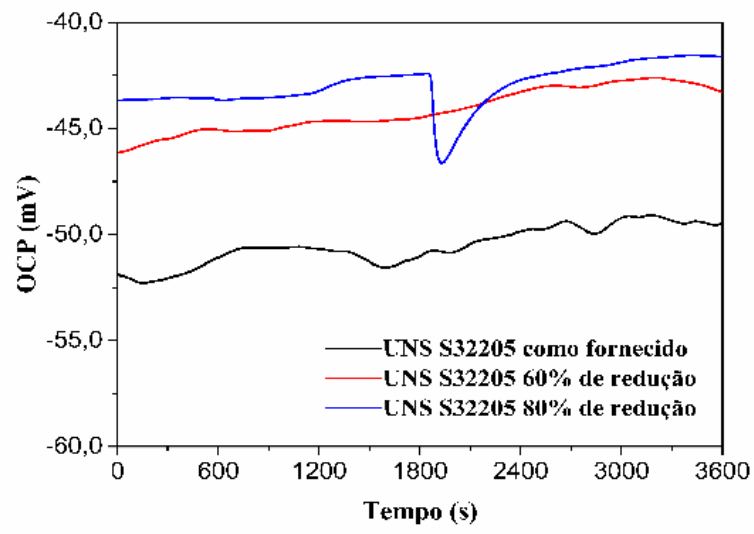

Figura 1. Potenciais de circuito aberto.

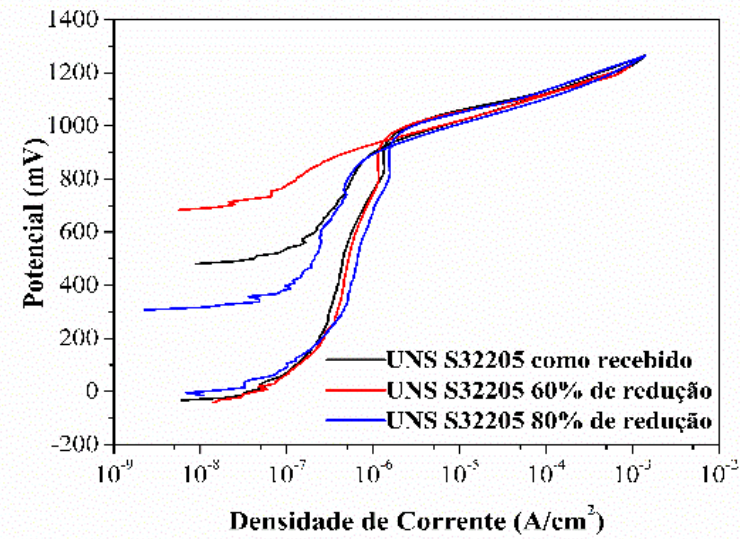

Figura 2. Curvas de polarização potenciodinâmica cíclica.

Tabela 2. Resultados experimentais médios

\begin{tabular}{lcccccc}
\hline Amostra & $\begin{array}{c}\text { Ep } \\
(\mathbf{m V})\end{array}$ & $\begin{array}{c}\text { Desvio } \\
(\mathbf{m V})\end{array}$ & $\begin{array}{c}\text { Erep } \\
(\mathbf{m V})\end{array}$ & $\begin{array}{c}\text { Desvio } \\
(\mathbf{m V})\end{array}$ & $\begin{array}{c}\text { OCP } \\
(\mathbf{m V})\end{array}$ & $\begin{array}{c}\text { Desvio } \\
(\mathbf{m V})\end{array}$ \\
\hline $\mathrm{CF}$ & 987 & 10 & 912 & 39 & $-51,3$ & 9,0 \\
\hline $60 \%$ & 994 & 14 & 937 & 4 & $-43,9$ & 16,7 \\
\hline $80 \%$ & 1010 & 26 & 921 & 30 & $-42,1$ & 17,2 \\
\hline
\end{tabular}

A curva de polarização cíclica fornece informação útil sobre a faixa de potencial acima da qual o material é susceptível à corrosão por pites. A Figura 2 mostra que as amostras possuem um alto potencial de pite e uma ampla região de passivação que pode ser atribuída ao alto teor de $\mathrm{Mo}$ e $\mathrm{Ni}$ presente nas mesmas. Esses elementos exercem um papel fundamental na estabilização do filme passivo formado na superfície dessas ligas [13]. Isso é um indicativo da excelente resistência à corrosão das amostras em questão.

As três amostras apresentaram comportamento similar na polarização cíclica. $O$ potencial de circuito aberto das amostras de aço laminadas a morno com $60 \%$ e $80 \%$ de redução na espessura em solução de $\mathrm{NaCl} 3,5 \% \mathrm{~m} / \mathrm{v}$ foi de $-43,9$ e $-42,1$ $\mathrm{mV}$, respectivamente. Para o aço como fornecido um potencial mais baixo foi observado, $-51,3 \mathrm{mV}$. As correntes de passivação para todas as amostras se encontram na mesma faixa, em torno de $10^{-6} \mathrm{~A} / \mathrm{cm}^{2}$.

Os potenciais de repassivação durante a polarização no sentido catódico também foram similares. Essa polarização (Fig. 2) mostrou uma pequena histerese positiva e 
um alto potencial de repassivação, que indica que o material possui capacidade de repassivação em solução de cloreto de sódio 3,5\%m/v.

Os valores de potencial de pite similares obtidos para as três amostras, sugerem que o tratamento termomecânico empregado não afetou de forma significativa a resistência à corrosão por pites do aço UNS S32205, porém esse potencial de pite característico não é o único parâmetro que deve ser usado para avaliar a resistência à corrosão por pite. Outro parâmetro importante é a densidade de pites (número de pites por área) [14].

As amostras foram observadas utilizando microscopia eletrônica de varredura após o teste eletroquímico (Fig. 3). Pequenos pites foram encontrados tanto na fase austenita quanto na fase ferrita, mostrando uma resistência à corrosão equivalente para as duas fases antes e após a laminação a morno. A corrosão por pite em solução salina confirmada pela Fig. 3 é consistente com os valores de OCP observados na Fig. 1. A corrosão por pites foi mais intensa na amostra CF, seguida pelas amostras com 60 e $80 \%$ de redução, como indicado pelo aumento do número de pites na Fig. $3(a-c)$.

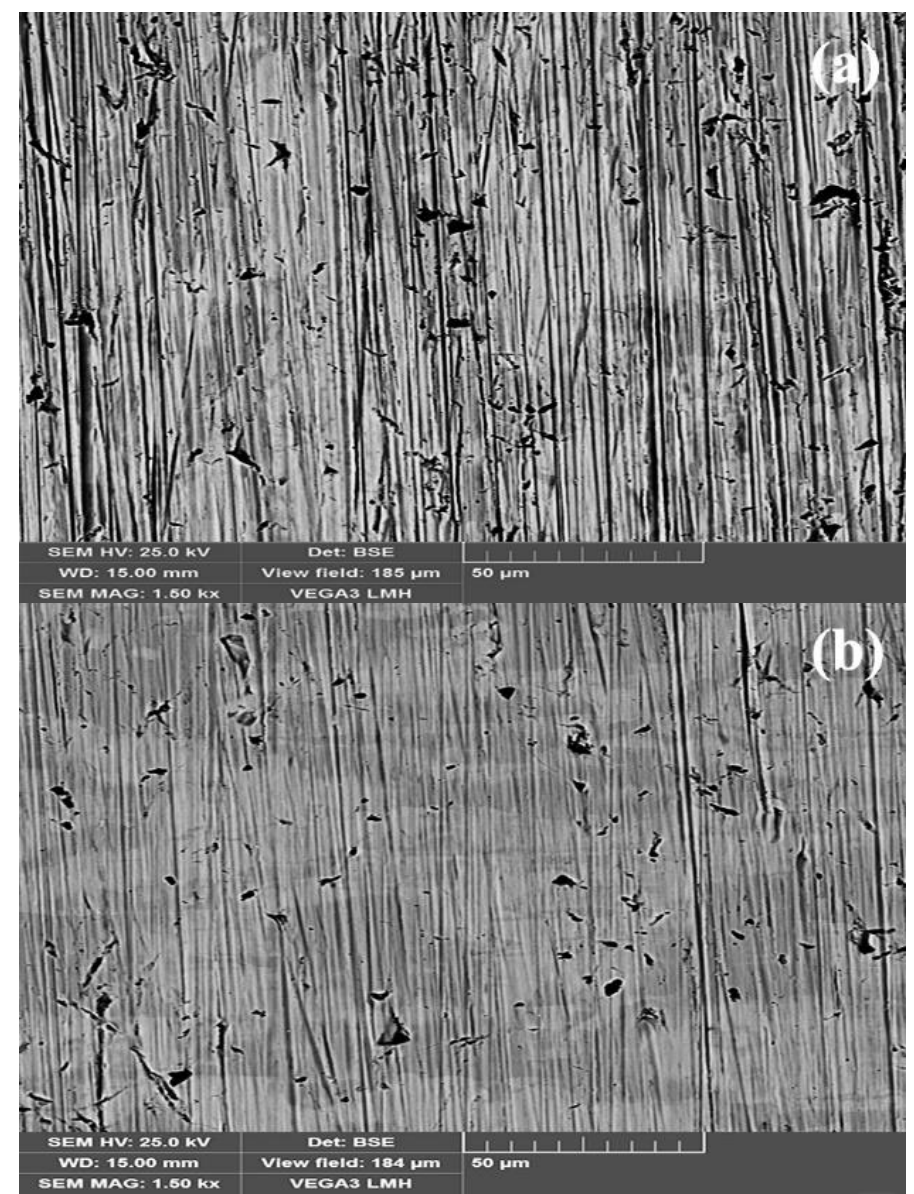




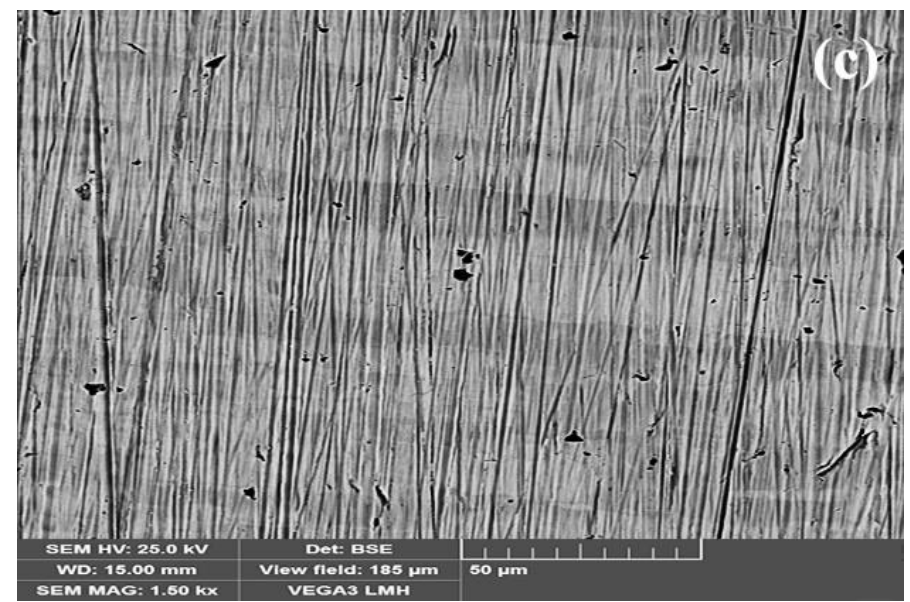

Figura 3. Morfologia dos pites após os testes de polarização das amostras (a) CF, (b) $60 \%$ de redução e (c) $80 \%$ de redução.

\section{CONCLUSÕES}

As amostras do aço inoxidável duplex, UNS S32205, apresentaram uma excelente resistência à corrosão em solução de $\mathrm{NaCl} 3,5 \%$. As mesmas mostraram capacidade de repassivação em meio contendo cloretos. Apesar da existência de certa similaridade entre os valores de potencial de circuito aberto e potenciais de pites obtidos para todas as amostras, o número de pites observado foi maior na amostra CF, seguida pelas amostras com 60 e $80 \%$ de redução.

\section{Agradecimentos}

Os autores agradecem a Aperam South America pelo fornecimento do aço de estudo. Também agradecem a CAPES-PROEX-PPGEM/UFMG, ao CNPq e a FAPEMIG pela concessão de bolsas de IC e auxílio financeiro.

\section{REFERÊNCIAS}

1 Escobar JD, Velásquez E, Santos TFA, Ramirez AJ, López D. Improvement of cavitation erosion resistance of a duplex stainless steel through friction stir processing (FSP). Wear. 2013;297(1-2):998-1005.

2 Noble DN. Metals handbook: welding, brazing, and soldering. Metals Park: ASM, 1993.

3 Sicupira DC, Cardoso Junior R, Bracarense AQ, Frankel GS, Lins VFC. Cyclic Polarization Study of Thick Welded Joints of Lean Duplex Stainless Steel for Application in Biodiesel Industry. Materials Research. 2017;20(1):161-167.

4 Jinlong L, Tongxiang L, Chen W, Limin D. Effect of ultrafine grain on tensile behaviour and corrosion resistance of the duplex stainless steel. Materials Science and Engineering C. 2016;62:558-563.

5 Ahmed MZ, Bhattacharjee PP. Microstructure, Texture, and Tensile Properties of a Severely Warm-Rolled and Annealed Duplex Stainless Steel. Steel Research International. 2016;87(4):472-483.

6 Chen L, Tan H, Wang Z, Li J, Jiang Y. Influence of cooling rate on microstructure evolution and pitting corrosion resistance in the simulated heat-affected zone of 2304 duplex stainless steels. Corrosion Science. 2012;58:168-174.

7 Stergiou V, Papadimitriou GD. Effect of an electron beam surface treatment on the microstructure and mechanical properties of SAF 2205 joints produced with electron beam welding. Journal of Materials Science. 2011;47(5):2110-2121. 
8 Gholami M, Hoseinpoor M, Moayed MH. A statistical study on the effect of annealing temperature on pitting corrosion resistance of 2205 duplex stainless steel. Corrosion Science. 2015;94:156-164.

9 Moura VS, Lima LD, Pardal JM, Kina AY, Corte RRA, Tavares SSM. Influence of microstructure on the corrosion resistance of the duplex stainless steel UNS S31803. Corrosion Science. 2008;59(8):1127-1132.

10 Lenard JG. Primer on Flat Rolling. 2nd ed. Waterloo: Elsevier; 2014.

11 Bhattacharjee PP, Zaid M, Sathiaraj GD, Bhadak B. Evolution of Microstructure and Texture During Warm Rolling of a Duplex Steel. Metallurgical and Materials Transactions A: Physical Metallurgy and Materials Science. 2013;45(4): 2180-2191.

12 ASTM G61-86. Standard test method for conducting cyclic potentiodynamic polarization measurements for localized corrosion susceptibility of iron-, nickel-, or cobalt-based alloys. West Conshohocken: ASTM International; 2003.

13 Blasco-Tamarit E, Igual-Munõz A, Anton JG, García-García D. Effect of aqueous LiBr solutions on the corrosion resistance and galvanic corrosion of an austenitic stainless steel in its welded and non-welded condition. Corrosion Science. 2006;48(4):863-886.

14 McCafferty E. Introduction to Corrosion Science. Springer; 2010. 